\title{
BMJ Open Young adult perceptions of JUUL and other pod electronic cigarette devices in California: a qualitative study
}

\author{
Emily Keamy-Minor, Julia McQuoid, Pamela M Ling
}

To cite: Keamy-Minor E, McQuoid J, Ling PM. Young adult perceptions of JUUL and other pod electronic cigarette devices in California: a qualitative study. BMJ Open 2019;9:e026306. doi:10.1136/ bmjopen-2018-026306

- Prepublication history for this paper is available online. To view these files, please visit the journal online (http://dx.doi. org/10.1136/bmjopen-2018026306).

Received 28 August 2018 Revised 24 December 2018 Accepted 15 February 2019

Check for updates

(C) Author(s) (or their employer(s)) 2019. Re-use permitted under CC BY-NC. No commercial re-use. See rights and permissions. Published by BMJ.

Department of Medicine, University of California San Francisco School of Medicine, San Francisco, California, USA

Correspondence to

Dr Pamela M Ling;

Pamela.Ling@ucsf.edu

\section{ABSTRACT}

Objective Electronic cigarettes (e-cigarettes) which utilise prefilled 'pods' (pod devices) entered the US market in 2015. One brand, JUUL, captured more than half the e-cigarette market in early 2018, and the US Food and Drug Administration recently warned its manufacturer about adolescent uptake. This is the first qualitative study to describe distinct features of pod devices that appear to contribute to their popularity among young people.

Design Qualitative interview study of young adults who had used pod devices. Participants were recruited from Facebook, other social media, street recruitment and via snowball sampling.

Setting Participants were from California, with most from the San Francisco Bay Area.

Participants Young adults (aged 18-29 years) using multiple tobacco products (cigarettes, e-cigarettes and/ or smokeless tobacco) were recruited. Of the sample of 60 participants, 24 were included in this analysis: 10 who reported experience with pod devices and 14 who used other non-pod e-cigarette devices.

Results Ten participants had used a pod device in the past year. Of the pod device users, seven still used a pod device at the time of the interview and five did so daily. Nearly all $(n=9)$ pod device users smoked cigarettes in the past month; none were daily smokers. The 14 participants who used non-pod devices provided a point of comparison. Participants highlighted some distinct aspects of pod devices that facilitated use, including their aesthetic similarity to personal electronics, high levels of nicotine delivery with distinct psychoactive effects, more discreet and shorter duration use sessions, and greater social acceptability than more ostentatious non-pod e-cigarettes. Conclusions Pod devices' unique characteristics likely encourage pod device uptake among young people. Limitations on advertising in youth channels, flavours and distribution, and education about nicotine addiction may decrease initiation among young people and non-smokers.

\section{INTRODUCTION}

'Next generation' electronic cigarettes (e-cigarettes) that utilise flavoured 'pods' emerged in the US market in $2015 .{ }^{1}$ Pods are typically prefilled e-liquid cartridges inserted into a closed-system e-cigarette device. Sales of 'pod devices' have markedly increased; the most popular brand, JUUL, captured $32 \%$ of the e-cigarette market in 2017 and $49.5 \%$ by

\section{Strengths and limitations of this study}

Novel and timely topic with almost no published research to date.

- Qualitative study provides rich insights into why pod devices are becoming so popular among youth, complementing the cross-sectional survey data published to date.

- The sample, while limited, represents the 'leading edge' of the epidemic, as young adults are early adopters and influencers for adolescents and others.

- Limited number of mostly male participants who used pod devices from a single geographic area.

- Study was of polytobacco users; participants who used pod e-cigarette devices were not purposively sampled.

January 2018. ${ }^{23}$ Although JUUL was the first of these products, ${ }^{4}$ similar devices, including MyJet, My Von Erl, Phix and Suorin, ${ }^{5}$ are now available. Cigarette companies have begun producing pod devices: Imperial Tobacco purchased My Von Erl and launched it as a line extension of the blu electronic cigarette, called myblu, in early 2018 . $^{6}$

JUUL's rapid rise in popularity was accompanied by marketing on social media widely used by young people, including Twitter, Instagram and YouTube. ${ }^{4}$ While some argue pod devices and other non-pod e-cigarettes may encourage smoking cessation, ${ }^{7}$ these devices can also recruit youth and young adults to start nicotine use. ${ }^{8}$ It is important, therefore, to understand why pod devices have become popular among young people.

A 2017 report on e-cigarette marketing ${ }^{9}$ noted JUUL advertisements emphasised the product's 'high tech features' and flavours. ${ }^{10-12}$ Kavuluru and colleagues' analysis of JUUL messages on Twitter and Reddit found that brand mentions were associated with places frequented by young adults (eg, 'school', 'class', 'dorm') and youthful activities (eg, dressing in JUUL-themed costumes). ${ }^{3}$ Messages on these sites included discussions 
about concealing use and strategies for obtaining pods underage.

To our knowledge, no prior studies have explored young adults' perceptions and uses of pod devices compared with other tobacco products. This paper draws from semi-structured interviews with 24 young adult polytobacco users in California to understand: (1) How young adults perceive and use next generation pod devices and (2) How, if at all, their use of pod devices differs from that of conventional cigarettes and other non-pod e-cigarettes.

\section{METHODS}

\section{Study procedures}

Participants were recruited through ads on Facebook and Instagram and completed online eligibility screening. Eligible participants were 18-29 years old and used more than one tobacco product in the past month. All participants completed questionnaires about tobacco product use and sociodemographic characteristics prior to interviews.

Sixty-minute semi-structured, in-depth interviews were conducted primarily in person in university seminar rooms, with some by phone, and were audio recorded. Interview topics included use of different tobacco products, experiences and motivations for use, perceived benefits, and how and why different tobacco products were used in daily routines. Participants received a US $\$ 100$ gift card. Pseudonyms have been applied to protect participant confidentiality.

\section{Data analysis}

The initial coding scheme included product types, product use routines and product perceptions. The third author created the initial codebook after closely reading six transcripts and holding group discussions during data collection. The coding scheme was further refined in iterative team meetings with the first and second authors to accommodate emergent themes after the first 12 transcripts had been coded by the first author. The first author applied the final coding scheme to the rest of the transcripts. Transcripts were ranked in order of semantic richness. Excerpts from 18 of the richest transcripts regarding participants' e-cigarette use were read in tandem by the first and second author. From these in-depth readings, detailed memos regarding emerging categories of interest (eg, characteristics of different e-cigarette devices) were made. ${ }^{13}$ Four of these 18 transcripts contained pod device content. An additional six transcripts that had not been included in the tandem reading contained content regarding pod devices. These were also included in this analysis to supplement content on pod devices, for a total of 24 transcripts. Additional detailed memos were made by the first author describing and analysing relevant pod device content from these excerpts, which were discussed with the second author. Saturation regarding e-cigarette use was reached after 24 transcripts. The third author performed an independent reading of pod device excerpts. All three authors discussed the memos and excerpts, identifying the following themes regarding unique characteristics of pod devices: sensory experience, health effects, aesthetic appeal, convenience, nicotine delivery, contexts of use and social meanings.

\section{Participants}

This paper draws from 24 interviews with participants who were current e-cigarette users. Of the 24 interviews reported in this paper, 10 participants were current or former pod-device users and 14 were current non-pod e-cigarette users (eg small 'cigalike' devices resembling cigarettes, medium devices like 'vape pens' and large 'tank' or 'box mod' devices). The study was designed prior to the popularisation of pod devices, so did not specifically recruit pod device users or systematically assess pod devices in the interview guide.

The 10 pod-device using participants were $18-28$ years old and had used a pod device (JUUL or My Von Erl) in the past year. Seven still used a pod device at the time of the interview and, of these, five did so daily. Most current pod device users owned their device; the two non-daily users shared a friend's device. Similar to national patterns of young adult smoking, ${ }^{14} 15$ nearly all pod-device using participants smoked cigarettes in the past month $(n=9)$, but none were daily smokers (table 1 ).

\section{Patient and public involvement}

Research questions were informed by feedback from prior qualitative studies conducted with the target population of young adult tobacco users. ${ }^{16}$ Participants were not directly involved in the study design, recruitment and conduct of the study.

\section{RESULTS}

Many perceived benefits of pod devices were consistent with previous findings on e-cigarettes. ${ }^{13}{ }^{16-19}$ Participants noted pleasurable sensory experiences, including good taste and smell, a quickly dissipating scent unlike cigarettes and less noticeable throat irritation than from cigarettes. ${ }^{18}$ For example, Sarah used her JUUL because just, generally, it smells nice; it tastes good'. Khaled preferred non-pod e-cigarettes to cigarettes because of the sweet flavour.

Also consistent with perceptions of non-pod e-cigarettes, pod devices were considered less harmful to health than cigarettes. ${ }^{16}$ Adam started using a non-pod e-cigarette because he considered them to be better for his long-term lung health and short-term comfort:

There's no chest pain, like my throat is not kind of itching, dry all the time, and kind of like hurting.

Similarly, Ben noted that JUUL hurt his throat less than cigarettes, Brandon considered JUUL to be better for his 'lung capacity', and William believed that the device is 'less harmful than literally everything else'. Participants distinguished between 'real' smoke and 'vapour', 
Table 1 Participant characteristics and tobacco product use in past month

\begin{tabular}{|c|c|c|c|c|c|c|c|c|c|}
\hline \multirow{2}{*}{$\begin{array}{l}\text { Participant } \\
\text { pseudonym }\end{array}$} & \multirow[b]{2}{*}{ Gender } & \multirow[b]{2}{*}{ Age } & \multirow{2}{*}{$\begin{array}{l}\text { Pod device } \\
\text { brand }\end{array}$} & \multirow{2}{*}{$\begin{array}{l}\text { Current or } \\
\text { former pod } \\
\text { device use }\end{array}$} & \multirow{2}{*}{$\begin{array}{l}\text { Days of pod } \\
\text { device uset }\end{array}$} & \multicolumn{3}{|c|}{$\begin{array}{l}\text { Days of non-pod e-cigarette } \\
\text { use by device size } \neq\end{array}$} & \multirow{2}{*}{$\begin{array}{l}\text { Days of } \\
\text { cigarette use }\end{array}$} \\
\hline & & & & & & Small & Medium & Large & \\
\hline William & $M$ & 18 & JUUL & Current & 30 & 0 & 12 & 0 & 0 \\
\hline Sarah & $\mathrm{F}$ & 19 & JUUL & Current & 30 & 0 & 0 & 2 & 15 \\
\hline Brian & M & 21 & JUUL & Current & 30 & 0 & 0 & 0 & 4 \\
\hline Brandon & $M$ & 19 & JUUL & Current & 30 & 0 & 0 & 5 & 4 \\
\hline Ben & $M$ & 20 & JUUL & Current & 6 & 0 & 0 & 1 & 8 \\
\hline Isabelle & $\mathrm{F}$ & 21 & JUUL & Former & N/A & 2 & 10 & 0 & 12 \\
\hline Robert & M & 26 & JUUL & Former & N/A & 0 & 0 & 0 & 23 \\
\hline Liam & $M$ & 26 & My Von Erl & Current & 30 & 0 & 0 & 0 & 25 \\
\hline James & $M$ & 28 & My Von Erl§ & Current & 6 & 0 & 0 & 0 & 25 \\
\hline David & M & 23 & My Von Erl & Former & N/A & 0 & 0 & 30 & 15 \\
\hline John & M & 21 & $\mathrm{~N} / \mathrm{A}$ & $\mathrm{N} / \mathrm{A}$ & N/A & 30 & 30 & 30 & 25 \\
\hline Adam & $\mathrm{M}$ & 20 & $\mathrm{~N} / \mathrm{A}$ & $\mathrm{N} / \mathrm{A}$ & N/A & 0 & 0 & 15 & 25 \\
\hline Michael & M & 27 & $\mathrm{~N} / \mathrm{A}$ & N/A & N/A & 5 & 4 & 5 & 5 \\
\hline Emily & $\mathrm{F}$ & 23 & $\mathrm{~N} / \mathrm{A}$ & $\mathrm{N} / \mathrm{A}$ & N/A & 5 & 0 & 0 & 30 \\
\hline Khaled & M & 23 & $\mathrm{~N} / \mathrm{A}$ & $\mathrm{N} / \mathrm{A}$ & N/A & 0 & 0 & 10 & 30 \\
\hline Victoria & $\mathrm{F}$ & 28 & $\mathrm{~N} / \mathrm{A}$ & $\mathrm{N} / \mathrm{A}$ & N/A & 5 & 10 & 0 & 30 \\
\hline Ajay & $M$ & 28 & $\mathrm{~N} / \mathrm{A}$ & N/A & N/A & 0 & 3 & 3 & 15 \\
\hline Esther & $\mathrm{F}$ & 24 & $\mathrm{~N} / \mathrm{A}$ & $\mathrm{N} / \mathrm{A}$ & N/A & 3 & 0 & 30 & 0 \\
\hline Mathew & M & 24 & $\mathrm{~N} / \mathrm{A}$ & $\mathrm{N} / \mathrm{A}$ & N/A & 0 & 2 & 0 & 15 \\
\hline Luis & $\mathrm{M}$ & 24 & $\mathrm{~N} / \mathrm{A}$ & $\mathrm{N} / \mathrm{A}$ & N/A & 0 & 0 & 15 & 1 \\
\hline Josh & M & 24 & $\mathrm{~N} / \mathrm{A}$ & N/A & N/A & 22 & 0 & 30 & 30 \\
\hline Mark & M & 29 & $\mathrm{~N} / \mathrm{A}$ & $\mathrm{N} / \mathrm{A}$ & $\mathrm{N} / \mathrm{A}$ & 3 & 0 & 3 & 2 \\
\hline Samuel & M & 26 & $\mathrm{~N} / \mathrm{A}$ & $\mathrm{N} / \mathrm{A}$ & N/A & 0 & 0 & 3 & 7 \\
\hline Carlos & $M$ & 20 & $\mathrm{~N} / \mathrm{A}$ & $\mathrm{N} / \mathrm{A}$ & $\mathrm{N} / \mathrm{A}$ & 0 & 1 & 0 & 20 \\
\hline
\end{tabular}

*Three participants reported using pod devices in the past but were not currently using at the time of the interview.

†Participants reporting non-daily pod device use did not own their devices and instead shared with a friend.

†Participants were presented with descriptions and pictures of small, medium and large e-cigarettes. Small devices consisted of disposable and rechargeable 'cigalikes'. Medium devices consisted of vapour pens. Large devices contained refillable tanks and included 'box mods'. §articipant did not provide a brand name, but physical description of device and flavour options was consistent with My Von Erl. N/A, not applicable.

expressing that the latter was most likely better for their health. This was true even for participants who had not smoked cigarettes regularly prior to pod device use.

Participants' accounts of pod devices differed from those of other non-pod e-cigarettes in several notable ways. These included the unique aesthetic appeal of pod devices, ability to deliver nicotine at high concentrations and the convenience of using them quickly and discreetly. Contexts of use and social meanings also appeared to differ from non-pod e-cigarettes.

\section{Aesthetic appeal}

Pod devices resemble personal electronics, and charge via Universal Serial Bus (USB) cable. Participants described them as 'sleek' (Brian) and 'elegant looking' (James). Some participants who used pod devices compared the shape to that of a flash-drive:
Pick up a pen. You know like this USB flash-drive? It's like one of those but only a little bit smaller. (Brandon)

These devices can also be charged on a computer, reinforcing associations with electronic devices. One participant described how pod device use was linked to his friend's computer use:

If I'm at his house and we're just sitting on the couch watching something, he's always got his computer out, and [the My Von Erl] charges in a little connector thing. And you can just pick it up and then do it. (James)

Compared with modifiable e-cigarette 'box mods', pod devices are smaller and more 'user friendly'. One participant described the appeal of pod devices' simple design: 
I did pick up something called a My Von Erl, which is smaller than this tape recorder. [...] It's like little pods $[\ldots]$. And you stick it in. And, like, it's really simple. (David)

In contrast, Michael found it difficult to bring his box mod with him when going out in the evening, noting that 'it's a really big thing to carry around, you know?'

Lastly, one participant's experience trying JUUL seemed to reflect new technology early adoption; it was exciting at first, but this novelty wore off:

I mean honestly, it's like a new car. You know, first few years are great. It's a newer car than most others. I'm talking a brand new car. Like you get it right off the lot. It's got like ten miles on it, right? And then a few years later, it's not new anymore. It's not interesting. $[\ldots][\mathrm{I}] \mathrm{t}$ kind of loses its value as being new and fun and exciting. (William)

\section{Nicotine delivery}

Although My Von Erl pods were sold in four different nicotine levels at the time of data collection $(0,9,18$ and $36 \mathrm{mg} / \mathrm{mL}$ ), JUUL pods were available only in $59 \mathrm{mg}$ / $\mathrm{mL}$ per pod. ${ }^{20}$ Discussion of nicotine content for My Von Erl was limited. Participants using JUUL for more than a month (Brandon, Sarah, Brian), noted JUUL's high concentration of nicotine. Brian described JUUL's standardised pods as 'ridiculously strong'. This was a marked departure from his box mod, which was 'all about the clouds and less about, like, actually getting that buzz'. Brandon started his description of JUUL with the observation, 'It's like a lot of nicotine, from what I understand'.

In addition to identifying JUUL's nicotine content, participants also described physical sensations likely attributable to nicotine delivery. One participant (William) described the JUUL sensation as 'a minute of just complete relaxation'. Others described triggers for JUUL similar to smoking triggers, such as following meals (Sarah) or after exercise (Brandon). A few participants experienced reduced cravings for nicotine (Liam). Sarah described a reduced desire to smoke cigarettes after daily use of JUUL: 'Like the less you're using cigarettes, the less appealing they become to you'.

This similarity to cigarettes in terms of sensation and nicotine delivery was something that Ajay said was missing from his 'vape pen'. During an attempt to switch from cigarettes to his friend's vape pen, he found that the vape pen had 'just kind of like a little different feel' compared with cigarettes. He credits this difference with preventing him from substituting products entirely, instead leading him to dual use of both cigarettes and a vape pen.

While some pod device users seemed to seek nicotine, others were concerned about addiction. Given JUUL's single nicotine concentration, some participants found it difficult to taper or reduce their nicotine intake, a strategy favoured by Brian during past cessation attempts with a box mod:
I don't want to be addicted to [nicotine], and the best way to quit would be-or the best way for me to quit would be to control it. And to control it, I think it's a lot easier with an e-cigarette that isn't the JUUL. The JUUL you can't control shit, but the, like, box mod you actually can. (Brian)

A tapering strategy was also preferred by two participants who used non-pod e-cigarettes. Luis enjoyed being able to control how much nicotine was in his box mod liquid. Josh had 'drawn back' from 12 to $6 \mathrm{mg} / \mathrm{mL}$ in his box mod, because he no longer felt like he needed as much nicotine.

Some pod device users were confident in their ability to avoid dependence by imposing limits on use. For example, William reported using JUUL in the morning to 'get the most effect', which might be analogous to smoking shortly after waking, an indicator of nicotine addiction. ${ }^{21}$ Nevertheless, he considered himself to be at low-risk for addiction:

You know, the only part of it is [sic] that is concerning is the nicotine usage and I'm very, very responsible. So, I didn't really see myself getting addicted needless to say. (William)

The nicotine level in pod devices appeared to influence the frequency and duration of participants' use in everyday life. Sarah reported taking fewer puffs at a time when using her JUUL (puffs per session) than she does with cigarettes. However, she said that she uses her JUUL at more points throughout the day (sessions per day):

But when I have the JUUL, it's honestly-each hit is a lot stronger, so it's like I'm going to be sitting there for like ten seconds and using it. [...] But then I'm going to want to use it again, because I didn't get the whole same amount of nicotine [as compared to a cigarette]. (Sarah)

Brian also took fewer puffs at a time when using his JUUL than with his box mod:

[I] t's just, like, I would die if I used it all day. I don't feel the need to, like, constantly be hitting it if I have it on me. (Brian)

In contrast, David noted that use of non-pod e-cigarettes can differ significantly from person to person:

I know people who, you know, like, will hit their vape once. And I know people who don't breathe oxygen, right? Like, they only use their vape.

Most participants reported that they typically used their pod device for fewer than $3 \mathrm{~min}$ at a time, with some taking up to a dozen hits over several minutes, and others taking several consecutive hits in under a minute. These variations in use resulted in most participants gauging use by how long a cartridge lasted, varying from 4 to 5 days (light use) to 1 day (heavy use). 
In comparison, use patterns of non-pod e-cigarettes were more difficult for participants to quantify. Compared with cigarettes, Khaled says that use of his box mod is 'harder to gauge':

I think it takes me a little longer [than with a cigarette], more puffs maybe until I'm like, 'Okay, I'm satisfied. I'll put this thing down now'.

\section{Convenience}

Pod devices are small, light and highly portable. The closed pods are tidier than some refillable devices, which can leak e-liquid. When David used a box mod, he found the device to be 'troublesome, because, like, the juice can get places'. He also frequently had to replace broken parts. Compared with non-pod e-cigarettes, he considered pod devices to be more 'pocketable'.

Pod devices also produce smaller aerosol clouds, allowing discreet use in public places. Liam's decision to switch from a box mod to a My Von Erl was partially motivated by a desire to be 'as discreet as possible':

'So, ultimately, [the box mod] was just, like, too bulky. It was, like-it's a really big-like, a really big device. And so, yeah, just it wasn't easy to carry around, and it just, like, the smoke output or the vapor output was just, like-just too, too much, just a little too crazy'.

(Liam)

Other participants liked the idea of discretion, even if they did not take advantage of this feature. Brandon noted that JUUL could be used discreetly 'if you needed to'. In contrast, lack of discretion was a significant concern for participants who used non-pod devices. As Esther explained of her box mod:

I don't want to be in public smoking these huge vapor clouds, but I know that I am going to get nicotine cravings. (Esther)

Ben reported that his friends had concealed their use of JUUL on airplanes. The speedy and intense nicotine delivery of JUUL (noted above), facilitated taking a quick 'hit' between activities (Sarah), enabling integration of pod device use more conveniently into the flow of the day.

\section{Contexts of use}

Participants described using pod devices in places and situations similar to other e-cigarettes, ${ }^{13} 2223$ such as indoors at home and in cars, while relaxing with friends, after eating and on breaks from work. Compared with cigarettes, both non-pod and pod e-cigarette devices are easy to use indoors, as they are not perceived to leave a 'lingering smell'. As Victoria, who uses a vape pen, explained:

You know if somebody has smoked a cigarette in a house. You know if it's been in a car. Even if it's been a while, there's that lingering smell. This [vape pen], it doesn't smell at all.
The absence of a tell-tale odour allowed Esther to use her box mod indoors without worrying about her landlord's no-smoking policy, and let Khaled feel safe in the knowledge that 'it's not going to piss roommates off'. Similarly, participants who used pod devices enjoyed doing so indoors. James used his friend's My Von Erl when relaxing on his couch. Brandon preferred JUUL to cigarettes, in part because 'you can pretty much do it inside. And it's not like you'll smell up a room with tobaccowith like traditional tobacco smell'. They also noted minimising use around adults and strangers. However, there were some indications that pod devices may be informally permissible in spaces where tobacco use is prohibited. This may be due to the small device size and minimal aerosol cloud. For example, unlike smoking, Sarah was allowed to use her JUUL indoors at her catering job even when she was not on break:

I usually just have my JUUL on hand, and if I'm ever in a behind-the-scenes setting, I'm allowed to just use that. So I'll just continuously use that throughout my work shift. (Sarah)

\section{Social meanings}

Pod devices appear to share many of the same social meanings as other e-cigarettes, especially when contrasted to cigarettes. Social meanings here refer to broader understandings of the social significance of using e-cigarettes, including social acceptability. ${ }^{24}$ Compared with cigarettes, both non-pod and pod devices are seen as more acceptable to use in most social contexts. This is likely related to participants' perceptions that they produce a more pleasant smell and are less harmful to health. Luis, who uses a box mod, indicated that people are 'less bothered by vaping, because the smell isn't the cigarette smell'. John explained that he is less worried about generating secondhand smoke with his non-pod e-cigarette than with cigarettes:

I take into consideration when I smoke because I don't want to affect others' health. So, pretty much in public areas where there tends to be a lot of children, I don't smoke [cigarettes]. Whereas, with the vaporizer, I feel like I'm able to smoke [vape] anywhere.

However, some participant accounts demonstrated that pod devices may have instigated a shift in the social meanings associated with using e-cigarettes towards even greater acceptability. While large box mods can evoke stereotypes of ostentation or disrespect, ${ }^{13}$ pod devices may not be subject to this stigma. Ajay, who uses a vape pen and occasionally a box mod, provided a vivid description of the social disapprobation associated with using non-pod e-cigarettes:

Like twoyears ago if you were out vaping we would just make fun of you the whole time we were smoking. And we'd just call you like you little sissy. Just smoke real cigarettes. 
James, who used pod devices, voiced similar criticisms of large box mods, calling them 'pretty weird' and 'kind of lame'. In comparison, pod devices are 'just really well accepted' among his friends. Brian contrasts the obnoxiously performative connotation of non-pod e-cigarettes with use of pod devices:

[S]o I went and bought an e-cigarette, and then I felt really awkward using e-cigarettes 'cause they're douchey, and so I got a JUUL because JUULs, for some reason, aren't douchey. (Brian)

The proliferation of pod devices throughout participants' social networks further highlights social acceptability. Most participants reported commonly sharing and using with friends. By the time Brandon first tried JUUL, half of his friends had already purchased their own device. Sarah and her best friend purchased JUULs at the same time, intending to switch to JUUL from cigarettes together.

\section{DISCUSSION}

This paper is the first to report on young adults' perceptions and uses of pod devices as compared with non-pod e-cigarettes in a way that may explain their popularity. We found that pod devices share many similarities with other e-cigarettes, including their desirable taste, smell and perceived health benefits. ${ }^{16-19} 22$ However, several distinguishing features were also evident. Participants enjoyed the 'tech appeal' aesthetic and associated pod devices with user-friendly personal electronics. ${ }^{9}$ JUUL pods were perceived to deliver a high dose of nicotine, enabling shorter consumption sessions. Pod devices' portability and discreet clouds made them particularly convenient. These characteristics appear to lend pod devices to inconspicuous use in a wide variety of formally prohibited tobacco use contexts, including in rental units and at workplaces. Finally, pod devices may have begun to reduce the social stigma previously associated with large 'box mod' devices.

Some characteristics of pod devices may help explain their appeal to younger populations. Associations with friendly and ubiquitous personal electronic devices rather than deadly and stigmatised tobacco products may facilitate use by young people, including non-smokers. These characteristics, paired with social media advertising, may encourage uptake among the young, particularly as social media content facilitates modelling and normalises use. ${ }^{34}$ A systematic review of JUUL marketing content from 2015 to 2018 on Twitter, Instagram and YouTube ${ }^{4}$ revealed an innovative, engaging and wide-reaching campaign conducted by JUUL and its affiliated marketers on these social media platforms, with audiences that disproportionately consisted of youth and young adults.

Use of pod devices by youth and non-smokers is particularly concerning as many participants readily spoke of strong nicotine delivery. Descriptions of situational triggers and early morning use suggests a risk of developing (perhaps unrecognised) nicotine dependence. Education about signs of nicotine addiction may also deter experimentation or motivate users to quit. Removal of flavours, offering reduced nicotine strength pods, restricting advertising in youth media channels and limiting distribution of pod devices might reduce uptake by youth and non-smokers. On the other hand, powerful nicotine delivery may facilitate pod devices' substitution for cigarettes, and some pod users spoke of decreased cigarette craving. The efficacy of pod devices for smoking cessation should be examined in randomised clinical trials; none have been published to date.

These findings also suggest several strategies for future research. Discrepancies between e-cigarette prevalence and sales ${ }^{4}$ suggest that pod devices may be inadequately captured on existing surveillance instruments perhaps due to distinct appearance, brands and terminology for use (eg, 'JUULing'). ${ }^{3}$ As the number and variety of nicotine products proliferate, measures to capture total nicotine intake and dependence need to be developed and should include novel nicotine delivery devices. Pod device use patterns and experiences should be examined separately from other non-pod e-cigarettes. Triangulating market research and epidemiological surveillance data may better characterise novel product uptake by different audiences; surveillance measures need to evolve as quickly as the e-cigarette market.

\section{Limitations}

This study did not specifically recruit pod device users, so the number of pod device using participants was small. Our pod device using participants were predominantly male. They could also be considered early adopters of pod devices, having begun use within the first 2 years after these products appeared on the market. The experiences of other young adults, particularly women and those who may be less prone to early adoption of new technology, may differ from the accounts presented here. However, pod using participants were able to provide rich descriptions of their products and how they used them. The accounts provided here reflect early 2017 pod devices, and do not include planned changes in the nicotine strengths in JUUL pods ${ }^{25}$ or newer refillable pod devices. ${ }^{26}$ Further, our analysis did not differentiate between young adults who were using pod devices as a cigarette cessation aid and those who were never established smokers. Finally, this cross-sectional analysis cannot determine whether participants progressed to cigarette use or were successful in sustaining a smoking cessation attempt. The differences in uses and perceptions between young adults who used pod devices to quit smoking with those who had never smoked prior to pod device initiation should be examined in future.

\section{CONCLUSION}

The tobacco product landscape is rapidly changing. Pod devices appear to have catalysed a shift in how many 
young adults perceive and use tobacco and nicotine. Pod devices' associations with personal electronics, efficient nicotine delivery, appealing flavour and odour, convenience and greater social acceptance encourages uptake and use by young adults. As companies promoting new e-cigarette devices replicate these product features and utilise youth media for promotion, these devices are likely to continue to be rapidly adopted by young people. Limits on advertising, availability and abuse liability of pod devices, and public education to counter industry messages normalising nicotine use may counteract these trends.

Contributors EK-M contributed to data collection, coded all transcripts, wrote analytical memos and led manuscript writing. JM contributed to study design, data collection, analytical framework and contributed to manuscript writing. PML obtained funding and contributed to study conception, design, data collection and manuscript writing. All authors contributed to data analysis, critical revision of the manuscript and approved the final manuscript for submission.

Funding Research reported in this study received funding from the National Cancer Institute grants R01-CA141661 and T32-CA113710. The content is solely the responsibility of the authors and does not necessarily represent the official views of the National Institutes of Health.

Competing interests None declared.

Patient consent for publication Obtained.

Ethics approval Ethics approval for this study was granted by the University of California, San Francisco Institutional Review Board.

Provenance and peer review Not commissioned; externally peer reviewed.

Data sharing statement Due to participant confidentiality, data are not available to the public for sharing.

Open access This is an open access article distributed in accordance with the Creative Commons Attribution Non Commercial (CC BY-NC 4.0) license, which permits others to distribute, remix, adapt, build upon this work non-commercially, and license their derivative works on different terms, provided the original work is properly cited, appropriate credit is given, any changes made indicated, and the use is non-commercial. See: http://creativecommons.org/licenses/by-nc/4.0/.

\section{REFERENCES}

1. Levy A. E-cigarette maker JUUL is raising $\$ 150$ million after spinning out of vaping company. 2017 https://www.cnbc.com/2017/12/19/ juul-labs-raising-150-million-in-debt-after-spinning-out-of-pax.html.

2. Robinson M. How a startup behind the 'iPhone of vaporizers' reinvented the e-cigarette and generated \$224 million in sales in a year. $2017 \mathrm{http}: / / w w w . b u s i n e s s i n s i d e r . c o m / j u u l-e-c i g a r e t t e-o n e-$ million-units-sold-2017-11.

3. Kavuluru R, Han S, Hahn EJ. On the popularity of the USB flash drive-shaped electronic cigarette Juul. Tob Control 2019;28:tobaccocontrol-2018-054259.

4. Huang J, Duan Z, Kwok J, et al. Vaping versus JUULing: how the extraordinary growth and marketing of JUUL transformed the US retail e-cigarette market. Tob Control 2019;28.
5. Hybels A. New, smaller vaping devices popular with teens harder for parents and schools to detect. $2018 \mathrm{http} / / \mathrm{www} . \mathrm{abc} 12 . \mathrm{com} /$ content/news/Smaller-devices-make-teen-vaping-harder-to-detect482950451.html.

6. Evans S. Imperial Brands launches new vaping product in USA. 2018 http://www.imperialbrandsplc.com/Media2/Key-announcements/ key-announcements/imperial-brands-launches-new-vaping-productin-usa.html.

7. Brown J, Beard E, Kotz D, et al. Real-world effectiveness of e-cigarettes when used to aid smoking cessation: a cross-sectional population study. Addiction 2014;109:1531-40.

8. Glantz SA, Bareham DW. E-cigarettes: use, effects on smoking, risks, and policy implications. Annu Rev Public Health 2018;39:215-35.

9. Cantrell J, Ganz O, Emelle B, et al. Mobile marketing: an emerging strategy to promote electronic nicotine delivery systems. Tob Control 2017;26:e1-e3.

10. Villanti AC, Johnson AL, Ambrose BK, et al. Flavored tobacco product use in youth and adults: findings from the first wave of the PATH study (2013-2014). Am J Prev Med 2017;53:139-51.

11. Thrasher JF, Abad-Vivero EN, Barrientos-Gutíerrez I, et al. Prevalence and correlates of E-cigarette perceptions and trial among early adolescents in Mexico. $J$ Adolesc Health 2016;58:358-65.

12. Pepper JK, Ribisl KM, Brewer NT. Adolescents' interest in trying flavoured e-cigarettes. Tob Control 2016;25:ii62-6.

13. McQuoid J, Keamy-Minor E, Ling PM. A practice theory approach to understanding poly-tobacco use in the United States. Crit Public Health 2018:1-16.

14. Sutfin EL, McCoy TP, Berg CJ, et al. Tobacco use by college students: a comparison of daily and nondaily smokers. Am J Health Behav 2012;36:218-29.

15. Schane RE, Glantz SA, Ling PM. Nondaily and social smoking: an increasingly prevalent pattern. Arch Intern Med 2009;169:1742-4.

16. Popova L, McDonald EA, Sidhu S, et al. Perceived harms and benefits of tobacco, marijuana, and electronic vaporizers among young adults in Colorado: implications for health education and research. Addiction 2017;112:1821-9.

17. Pokhrel P, Fagan P, Kehl L, et al. Receptivity to e-cigarette marketing, harm perceptions, and e-cigarette use. Am J Health Behav 2015;39:121-31.

18. Pokhrel P, Herzog TA, Muranaka N, et al. Young adult e-cigarette users' reasons for liking and not liking e-cigarettes: a qualitative study. Psychol Health 2015;30:1450-69.

19. Lucherini M, Rooke C, Amos A. E-cigarettes, vaping and performativity in the context of tobacco denormalisation. Sociol Health IIIn 2018;40:1037-52.

20. FAQS: JUULpods \& juice. https://support.juul.com/home/learn/faqs/ juulpod-basics.

21. Heatherton TF, Kozlowski LT, Frecker RC, et al. The Fagerström test for nicotine dependence: a revision of the Fagerström Tolerance Questionnaire. Br J Addict 1991;86:1119-27.

22. Pokhrel P, Herzog TA, Muranaka N, et al. Contexts of cigarette and e-cigarette use among dual users: a qualitative study. BMC Public Health 2015;15:859.

23. Keane H, Weier M, Fraser D, et al. 'Anytime, anywhere': vaping as social practice. Crit Public Health 2017;27:465-76.

24. Blue S, Shove E, Carmona C, et al. Theories of practice and public health: understanding (un)healthy practices. Crit Public Health 2016;26:36-50.

25. Tolentino J. The promise of vaping and the rise of JUUL. 2018.

26. Suorin Air - replacement pod. https://www.suorinusa.com/products/ suorin-air-replacement-pod?variant $=40150622349$. 